\title{
Orally Administration of Propranolol, Lorazepam, and Combination of Propranolol/Lorazepam, and Reducing Anxiety Before Surgery
}

\author{
Siamak Yaghoobi, ${ }^{1} \quad$ Behnam Mahmoodiyeh, ${ }^{2}$ Marzieh Beygom Khezri, ${ }^{3}$ Seyed \\ Mohammadreza Hashemian, ${ }^{4}$ and Alireza Jahangiri Fard,
}

${ }^{1}$ Shahid Rajaie Hospital, Qazvin University of Medical Sciences, Qazvin, IR Iran

${ }^{2}$ Tracheal Diseases Research Center, National Research Institute of Tuberculosis and Lung Diseases (NRITLD), Shahid Beheshti University of Medical Sciences, Tehran, IR Iran ${ }^{3}$ Kosar Hospital, Qazvin University of Medical Sciences, Qazvin, IR Iran

${ }^{4}$ Chronic Respiratory Diseases Research Center, National Research Institute of Tuberculosis and Lung Diseases (NRITLD), Shahid Beheshti University of Medical Sciences, Tehran, IR Iran 5 Lung Transplantation Research Center, National Research Institute of Tuberculosis and Lung Diseases (NRITLD), Shahid Beheshti University of Medical Sciences, Tehran, IR Iran

${ }^{*}$ Corresponding author: Alireza Jahangiri Fard, Lung Transplantation Research Center, National Research Institute of Tuberculosis and Lung Diseases (NRITLD), Shahid Beheshti University of Medical Sciences, Tehran, IR Iran. Tel: +98-9121820899, Fax:+98-2126109644, E-mail: alirezajahangiri@sbmu.ac.ir

Received: March 1, 2015; Accepted: March 20, 2015

Background: Anxiety before surgery is a frequent occurrence in patients. There are several strategies such as visiting the patient before surgery, reinforcing the patient's confidence through an intimate communication with a psychologist or giving sedation to minimize the surgery-related anxiety. Benzodiazepines and beta blockers are the most commonly used drugs to alleviate preoperative anxiety.

Objectives: The current study aimed to compare the effect of orally administered propranolol, lorazepam, and propranolol plus lorazepam on reducing patients' anxiety before surgery and also on hemodynamic changes before and during operation.

Patients and Methods: This double-blind clinical trial was carried out on 75 patients of both genders aged 18 - 45 years considered as being American Society of Anesthesiologists (ASA) Physical Class (PS) 1 and ASA PS 2 categories according to the American Society for anesthesiologists physical status classification system. Patients were randomly divided into three equal groups marked as group A (lorazepam $2 \mathrm{mg}$ ), group B (propranolol $20 \mathrm{mg}$ ), and group C (lorazepam $2 \mathrm{mg}$ plus propranolol $20 \mathrm{mg}$ ) who received the designated drug a night before the surgery. The degree of anxiety was assessed using the Amsterdam preoperative anxiety and information scale (APAIS). The level of anxiety, blood pressure, and heart rate were measured for all patients the night before surgery, on the day of surgery, and every five minutes from intubation time for a total of 15 minutes. Data analysis was performed by statistical tests including Anova and Chi-square test using SPSS version 15.

Results: The findings of the current study showed that all three drug groups could reduce the degree of anxiety however, lorazepam alone decreased anxiety more than the other two groups $(\mathrm{P}=0.001)$. Furthermore, propranolol alone or in combination with lorazepam showed similar effect on reducing systolic blood pressure and heart rate when entering the operating room and during surgery $(\mathrm{P}>0.05)$ whereas lorazepam alone had no significant effect on these hemodynamic parameters, statistically.

Conclusions: Considering the results obtained in the study, it was concluded that the combination of propranolol and lorazepam was a better premedication in patients with high stress who needed hemodynamic maintenance during surgery.

Keywords: Anxiety; Premedication; Propranolol; Lorazepam

\section{Background}

Anxiety is considered as a major complaint in majority of the patients who consult surgeons for a possible surgical procedure and also as an effective and preventing factor in patients' resistance to surgical procedures. Preoperative anxiety and its associated problems (severe changes in hemodynamic parameters, the occurrence of arrhythmia with occasional dangerous hemodynamic complications during anesthesia, agitation and low pain threshold following surgery, etc.) are well-known in the field of a surgeon and anesthetist's activity and the necessity to prevent it is the focus of numerous research projects (1). At present, the administration of oral or intramuscular pre-anesthetic to provide the patient with relaxation, reduce anxiety before anesthesia, lower an- esthetic dose to induce anesthesia, and recovery from anesthesia is very common (1). There are several drug regimens to reduce the adrenergic responses in patients, leading to decreased anxiety before surgical procedures. These compounds include benzodiazepines, $\alpha$-blockers such as droperidol, $\beta$-blockers such as propranolol and atenolol, low dose narcotics, and other drugs among which benzodiazepines and $\beta$-blockers such as propranolol are more common $(1,2)$.

Benzodiazepines are the most frequent drugs prescribed as premedication although they cause different anxiolytic effects. Lorazepam is a high potency benzodiazepine drug with short-term anxiety-reducing effect. It also has hypnotic, anticonvulsant, and amnesic proper-

Copyright (C) 2015, Shahid Beheshti University of Medical Siences. This is an open-access article distributed under the terms of the Creative Commons AttributionNonCommercial 4.0 International License(http://creativecommons.org/licenses/by-nc/4.0/) which permits copy and redistribute the material just in noncommercial usages, provided the original work is properly cited. 
ties with occasional respiratory depression and rare episode of hypotension (1). Propranolol is a nonspecific $\beta$-blockers with known cardiovascular effects which is used as an auxiliary drug to reduce anxiety through inhibition of sympathetic nerve stimulation and reduction of anxiety symptoms $(1,2)$. It is shown that propranolol is effective to treat stage fright and pathological anxiety with minimal sedation and least damage to psychomotor skills following administration of the drug. Low cost and oral route of administration makes propranolol a substitute for expensive preoperative drugs (3). There are numerous publications on assessing preoperative anxiety using different premedication and benzodiazepines and propranolol are two wellknown and effective drugs to fight the anxiety prior to a surgical procedure (4-8).

\section{Objectives}

The current study aimed to investigate the anxiolytic effect of orally administered propranolol, lorazepam, and a combination of propranolol and lorazepam on patients before surgery, and also to evaluate the hemodynamic changes that might happen following the administration of these drugs before and during general anesthesia.

\section{Patients and Methods}

The study was a double-blind clinical trial performed on patients undergoing minor to intermediate risk elective surgeries such as inguinal hernia repair, transurethral lithotomy, tibia fracture repair, were referred to Shahid Rajaie Hospital in Qazvin from March to August 2013. Patients were 18 - 45 years old with the American Society of Anesthesiologists (ASA) physical class I or II. Excluding criteria were the history of continuous consumption of benzodiazepines, anti-psychotics, alpha- and beta-blockers, narcotics, and antianxietics drugs, the history of panic attacks and other psychotic problems, congestive heart failure, asthma, chronic obstructive pulmonary disease (COPD), diabetes type I or II, hypertension, and allergic reaction to foods and drugs. Each patient was visited by an anesthesiologist resident the night before surgery and if the conditions to enter the study were confirmed, the patient was given the necessary explanations concerning the methodology of the study. Upon patient's verbal agreement to be included in the study, written consent was obtained, the questionnaire containing APAIS questions, blood pressure (BP) level, and heartbeat rate (HR) was completed and kept in patient's medical history. A total of 75 patients were randomly divided into three equal groups and marked as group A, B and C according to the card randomly selected by each patient, indicating the type of drug package each patient had to be delivered by a nurse while neither the anesthesiologist resident nor the nurse in charge of drug delivery were aware of the contents of the drug packages. The prescribed drug packages were package A (lorazepam $2 \mathrm{mg}+$ placebo), package $B$ (propranolol $20 \mathrm{mg}+$ placebo), and package $\mathrm{C}$ (propranolol $20 \mathrm{mg}+$ lorazepam $2 \mathrm{mg}$ ). In the present study, the APAIS anxiety scale was applied to evaluate the degree of anxiety in the patients. The APAIS anxiety scale consists of six questions, two associated with anesthesia related anxiety, two covering the surgical related anxiety, and the last two concerning the patient's need for the information on anesthesia and surgery stages. Patients were asked to give scores of 1 - 5 to the questions. The total scores were 30, indicating the occurrence of anxiety at its highest level. The APAIS anxiety scale was reevaluated for each patient before and immediately after the entry to the operative room and the results were recorded. Later, all patients were subjected to routine monitoring including $\mathrm{HR}$, $\mathrm{BP}$ and Electrocardiogram (ECG) with values recorded under general anesthesia using fentanyl $2 \mathrm{mg} / \mathrm{kg}$ and midazolam $0.2 \mathrm{mg} / \mathrm{kg}$ as premedication agents. The induction of anesthesia was performed by injection of sodium thiopental $5 \mathrm{mg} / \mathrm{kg}$ and atracurium $0.5 \mathrm{mg} / \mathrm{kg}$ followed by tracheal intubation and administration of isoflurane 102 minimum alveolar concentration (MAC) for the maintenance of anesthesia. Patients' HR and Systolic blood presure (SBP) were measured every five minutes from the beginning of surgery and continued for 15 minutes. Data were reexamined, transferred to the statistical software (SPSS version 15), and the comparison of observed outcomes was performed using statistical tests including the Chi-square and the Fisher exact tests for classified data and ANOVA for quantitative data while a $P$ value $\leq 0.05$ was considered significant.

\section{Results}

There was no significant age difference between the three study groups $(\mathrm{P}=0.1)$. Also, a similar gender difference was observed among the study groups $(P=0.7)$. The collected data showed that the mean anxiety in all three groups on the day of surgery was significantly different from that of the night before surgery; the mean anxiety in the three groups on the day of surgery was lower than that of the night before surgery $(\mathrm{P}<0.05)$. However, the degree of anxiety in the group receiving lorazepam was less than those of the other two groups revealing a significant difference between the three groups; although the difference between the lorazepam group and propranolol or lorazepam plus propranolol groups was significant, the difference between the propranolol and lorazepam plus propranolol groups was insignificant ( $P$ $=0.82$ ) (Table 1 ).

The obtained results also showed a significant difference in the mean systolic blood pressure on the day of surgery $(\mathrm{P}=0.004)$ and every five minutes from the beginning of anesthesia $(\mathrm{P}=0.001)$ between the three groups. The Tukey test revealed a significant difference in reduction of systolic blood pressure between the lorazepam 
group and propranolol and lorazepam plus propranolol groups whereas the difference between the propranolol group and propranolol plus lorazepam group was insignificant $(\mathrm{P}=0.692)$. No significant difference in the mean systolic blood pressure on the night prior to surgery was observed $(\mathrm{P}=0.22)$ (Table 2$)$.

There was no significant difference in the HR value on the night before the surgery among the three study groups $(P=0.06)$, but a significant difference in the HR value on the night before surgery and every five minutes from the beginning of surgery was observed among the three groups. The Tukey test showed a significant difference in the reduction of HR between the lorazepam group and propranolol and lorazepam plus propranolol groups whereas no statistically significant difference was observed between the propranolol group and lorazepam plus propranolol group $(\mathrm{P}=0.728)$ (Table 3$)$.

Table 1. Comparing Anxiety in the Three Study Groups According to the Amsterdam Preoperative Anxiety and Information Scale (APAIS) Anxiety Scale ${ }^{a}$

\begin{tabular}{|c|c|c|c|}
\hline Group & $\begin{array}{c}\text { Anxiety (on the Day of } \\
\text { Surgery) }\end{array}$ & $\begin{array}{c}\text { Anxiety (on the Night Before } \\
\text { Surgery) }\end{array}$ & P Value \\
\hline Lorazepam & $14.2 \pm 3$ & $17.8 \pm 4.3$ & 0.000 \\
\hline Propranolol & $16.8 \pm 3.2$ & $18.8 \pm 4.3$ & 0.002 \\
\hline Lorazepam/propranolol & $14.9 \pm 2.2$ & $17.7 \pm 3.9$ & 0.000 \\
\hline
\end{tabular}

${ }^{\mathrm{a}}$ The values are presented as Mean \pm SD.

Table 2. Comparing Mean SBPSystolic Blood Pressure on the Night and Day Before Surgery, and Every Five Minutes from the Beginning of Surgery in the Three Study Groups a

\begin{tabular}{|c|c|c|c|c|}
\hline \multirow{2}{*}{ Mean SBP ${ }^{b}$} & \multicolumn{3}{|c|}{ Group } & \multirow{2}{*}{ P Value } \\
\hline & Lorazepam & Propranolol & Lorazepam/Propranolol & \\
\hline $\begin{array}{l}\text { Every } 5 \mathrm{~min} \text { from the beginning of } \\
\text { anesthesia }\end{array}$ & $104 \pm 7.3$ & $98 \pm 6.7$ & $97 \pm 7.7$ & 0.000 \\
\hline On the day of surgery & $113 \pm 8.5$ & $107 \pm 8.0$ & $105 \pm 8.0$ & 0.004 \\
\hline On the night before surgery & $116 \pm 5.0$ & $115 \pm 7.0$ & $118 \pm 7.0$ & 0.226 \\
\hline
\end{tabular}

a The values are presented as Mean \pm SD.

b SBP, Systolic Blood Presure.

Table 3. Comparing Heartbeat Rate on the Night Before Surgery, on the Day of Surgery, and Every Five Minutes From the Beginning of Surgery in the Three Study Groups a

\begin{tabular}{lcccc}
\hline \multirow{2}{*}{ Mean HR } & \multicolumn{3}{c}{ Group } & P Value \\
\cline { 2 - 4 } & Lorazepam & Propranolol & Lorazepam/Propranolol & \multirow{2}{*}{0.001} \\
\hline Every 5 min after anesthesia induction & $72 \pm 7$ & $63 \pm 5$ & $64 \pm 4$ & 0.001 \\
\hline On the day of surgery & $76 \pm 5$ & $69 \pm 5$ & $70 \pm 6$ & 0.06 \\
\hline On the night before surgery & $76 \pm 6$ & $79 \pm 3$ & $76 \pm 6$ & \\
\hline
\end{tabular}

a Values are presented as Mean \pm SD. 


\section{Discussion}

The results of the current study indicated that lorazepam, propranolol, and the combination of lorazepam/ propranolol were effective to reduce the degree of anxiety before surgery, but the effect of lorazepam, compared to propranolol and the combination of lorazepam/propranolol was higher; although the difference in degree of anxiety reduction between the propranolol and lorazepam/propranolol groups was statistically insignificant.

Surgery-related anxiety is an unpleasant feeling which could lead to hemodynamic changes and affect the HR and BP during anesthesia, resulting in occurrence of cardiovascular damages throughout or after the surgery. Furthermore, anxiety could increase the need for anesthetic drugs; hence, the administration of anxietyreducing agents is recommended for all patients before surgical procedures (1). The anxiety-reducing effect of drugs such as benzodiazepines, narcotics, and betaand alpha-blockers are confirmed in many studies (9, 10). De Witte et al. showed that the oral administration of alprazolam and midazolam for the patients undergoing gynecological laparoscopic surgery considerably decreased the degree of anxiety before surgery (9). In another study by Dyck and Chung, the administration of oral diazepam and propranolol caused a similar decrease in the level of surgery-related anxiety and the speed in returning the patients' cognitive functioning following surgery was faster in propranolol group than the diazepam group and this might have been due to the light anesthesia in propranolol group and the result of receiving lower anesthetic drug (10).

There are numerous studies concerning the anxiety-reducing effect of benzodiazepines or propranolol alone $(3,5,11,12)$, but so far, to the best of the authors' knowledge, no study compared the effect of simultaneous administration of a benzodiazepine and propranolol on patients' anxiety before surgery. File and Lister compared the effect of propranolol and lorazepam on anxiety in normal student volunteers and concluded that both drugs produce the same effect on reducing the anxiety associated with intellectual stress (11). Since the changes in hemodynamic parameters of different patients during anesthesia could be accompanied by dangerous and potentially fatal outcomes, the present study also investigated the level of HR and BP in patients. Based on the current study data, lorazepam produced no statistically significant blood pressure-reducing effect in patients at the time of entry to the operating theatre and also during anesthesia whereas propranolol alone or in combination with lorazepam caused a reduction in the blood pressure of patients at the time of entry to operating theatre and during anesthesia. Therefore, it seems that the administration of propranolol is beneficial in reducing blood pressure and hemodynamic maintenance of patients throughout surgery.The effect of lorazepam in reducing the HR at the time of entry to operating room was not significant but in comparison with the primary HR and the mean HR during anesthesia, lorazepam produced a significant effect. This difference could be due to sympathetic inhibition and the patient's reduced anxiety following the initiation of anesthesia under the influence of anesthetic drugs as well as the anxiety-reducing effect of lorazepam. Both propranolol and propranolol/lorazepam groups produced similar effects on reducing the HR with no significant difference between the two groups, indicating that lorazepam was more effective than propranolol and propranolol/lorazepam in reducing the $H R$.

Hermann showed that the combination of $80 \mathrm{mg}$ propranolol and $1 \mathrm{mg}$ lorazepam was ineffective or caused clearance reduction and drug elimination; whereas propranolol, to some extent, prolonged the time for diazepam elimination and clearance reduction (13).

It could be concluded that the combination of propranolol and lorazepam, compared to propranolol alone, has no effect on hemodynamic maintenance of patients; although it acts better than lorazepam alone while the anxiety-reducing effect of this combination of drugs is not higher than that of lorazepam alone. Therefore, concerning the hemodynamic maintenance, it is recommended to administer propranolol for patients during surgical procedures. Propranolol alone, similar to other drugs, is equally effective in maintaining hemodynamic responses; however, when lorazepam is administered as premedication, it is suggested to be co-prescribed with propranolol to maintain hemodynamic stability of the patients during surgery.

Other parts of the present study showed that the degree of anxiety prior to surgery was higher in females than males and that the level of anxiety had no correlation with age and the education level of patients. The current study results, in this regard, were fully comparable with those of the other studies. To the authors' best knowledge, there is no similar study concerning the comparison of simultaneous effect of a benzodiazepine/beta-blocker combination on reducing the degree of anxiety in patients prior to surgery and its further impact on hemodynamic changes during anesthesia, which prevents the authors from giving a further comprehensive discussion regarding the entire data collected in the current study. It is suggested that in future studies the anesthetic drug doses, required for patients, be investigated in different groups. Also, assessing the sleep quality of patients could be another beneficial parameter in achieving a better drug combination with higher anxiety-reducing effect for patients prior to surgery. Likewise, comparative studies on administration of alpha-blockers versus betablockers and benzodiazepines could provide helpful information on administration of these drugs for patients before and during surgery. 


\section{Acknowledgements}

The authors would like to thank all colleagues at Rajaie Hospital that without their contribution the present work would not have been completed.

\section{Authors' Contributions}

Study concept and design: Siamak Yaghoobi; acquisition of data: Behnam Mahmoodiyeh, Marzieh Beygom Khezri; analysis and interpretation of data: Seyed Mohammadreza Hashemian; drafting of the manuscript: Alireza Jahangiri Fard.

\section{References}

1. Miller RD. Miller`s Anesthesia. 7th ed. Philadelphia: Churchill Lvingstone; 2008.

2. Miller RD. Anesthesia. 5th ed. Philadelphia: Churchill Livigsone; 2000.

3. Saha DK, Kader MA, Kamal MM, Akhtaruzzaman AKM, Iqbal KM. Effect of Low Dose Propranonol on Perioperative Stress Induced Hemodynamic Changes in Upper Abdominal Surgery. J Bangladesh Soc of Anaesthesiologists. 2006;19(1):14-9.

4. Liu SS, Wu CL. The effect of analgesic technique on postoperative patient-reported outcomes including analgesia: a systematic re- view. Anesth Analg. 2007;105(3):789-808.

5. Moshiri E, Yazdi B, Khalili M. The comparison effect of midazolam with propofol on pre-operative anxiolysis. Arak Med Univ J. 2009;12(1):87-94.

6. Vogele C. Hospitalisation and stressful medical procedures. Health Psychology. Malden, Mass, Oxford: BPS Blackwell; 2004. patients in hospital. pp. 288-304.

7. Ercan S. Relationship between psychological preparation, preoperative and postoperative anxiety, and coping strategies in children and adolescents undergoing surgery. Ankara: Middle East Technical University; 2003.

8. Caumo W, Hidalgo MP, Schmidt AP, Iwamoto CW, Adamatti LC, Bergmann J, et al. Effect of pre-operative anxiolysis on postoperative pain response in patients undergoing total abdominal hysterectomy. Anaesthesia. 2002;57(8):740-6.

9. De Witte JL, Alegret C, Sessler DI, Cammu G. Preoperative alprazolam reduces anxiety in ambulatory surgery patients: a comparison with oral midazolam. Anesth Analg. 2002;95(6):1601-6.

10. Dyck JB, Chung F. A comparison of propranolol and diazepam for preoperative anxiolysis. Can J Anaesth. 1991;38(6):704-9.

11. File SE, Lister RG. A comparison of the effects of lorazepam with those of propranolol on experimentally-induced anxiety and performance. BrJ Clin Pharmacol.1985;19(4):445-51.

12. Magarey JM. Propofol or midazolam--which is best for the sedation of adult ventilated patients in intensive care units? A systematic review. Aust Crit Care. 2001;14(4):147-54.

13. Ochs HR, Greenblatt DJ, Verburg-Ochs B. Propranolol interactions with diazepam, lorazepam, and alprazolam. Clin Pharmacol Ther. 1984;36(4):451-5. 